\section{Brain Cortical Activation during Imagining of the Wrist Movement Using Functional Near Infrared Spectroscopy (fNIRS)}

\author{
Maziar Jalalvandi ${ }^{\oplus}$, Nader Riyahi Alam ${ }^{2,3 * \odot}$, Hamid Sharini4, \\ Hasan Hashemi ${ }^{5,6}$, Mohadeseh Nadimi ${ }^{7}$
}

\begin{abstract}
Background: fNIRS is a useful tool designed to record the changes in the density of blood's oxygenated hemoglobin (oxyHb) and deoxygenated hemoglobin (deoxyHb) molecules during brain activity. This method has made it possible to evaluate the hemodynamic changes of the brain during neuronal activity in a completely non-aggressive manner.

Objective: The present study has been designed to investigate and evaluate the brain cortex activities during imagining of the execution of wrist motor tasks by comparing fMRI and fNIRS imaging methods.
\end{abstract}

Material and Methods: This novel observational Optical Imaging study aims to investigate the brain motor cortex activity during imagining of the right wrist motor tasks in vertical and horizontal directions. To perform the study, ten healthy young right-handed volunteers were asked to think about right-hand movements in different directions according to the designed movement patterns. The required data were collected in two wavelengths, including 845 and 763 nanometers using a 48 channeled fNIRS machine.

Results: Analysis of the obtained data showed the brain activity patterns during imagining of the execution of a movement are formed in various points of the motor cortex in terms of location. Moreover, depending on the direction of the movement, activity plans have distinguishable patterns. The results showed contralateral M1 was mainly activated during imagining of the motor cortex $(p<0.05)$.

Conclusion: The results of our study showed that in brain imaging, it is possible to distinguish between patterns of activities during wrist motion in different directions using the recorded signals obtained through near-infrared Spectroscopy. The findings of this study can be useful in further studies related to movement control and BCI.

Citation: Jalalvandi M, Riyahi Alam N, Sharini H, Hashemi H, Nadimi M. Brain Cortical Activation during Imagining of the Wrist Movement Using Functional Near Infrared Spectroscopy (fNIRS). J Biomed Phys Eng. 2021;11(5):583-594. doi: 10.31661/jbpe.v0i0.1051.

\section{Keywords}

Hemodynamics; Near-Infrared; Motor Cortex; Functional Neuroimaging

\section{Introduction}

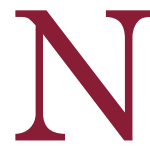

ear-infrared spectroscopy (fNIRS) is an effective and indirect method measuring of blood hemodynamic changes concerning a specific activity. According to the neurological principles, this neurological activity and vascular responses are interconnected called neuromuscular coupling. In this method, infrared light is transmitted to the surface of the skull, and the absorption spectrum of the light ab-
${ }^{1} \mathrm{MSc}$, Department of Medical Physics and Biomedical Engineering, School of

Medicine, Tehran University of Medical Sciences (TUMS), Tehran, Iran

${ }^{2} \mathrm{PhD}$, Department of Medical Physics and Biomedical Engineering, School of

Medicine, Tehran Univer-

sity of Medical Sciences

(TUMS), Tehran, Iran

${ }^{3} \mathrm{PhD}$, Medical Pharmaceu-

tical Sciences Research

Centre (MPRC), The

Institute of Pharmaceutical

Sciences, Tehran Univer-

sity of Medical Sciences,

Tehran, Iran

${ }^{4} \mathrm{PhD}$, Department of Medi-

cal Physics and Biomedical

Engineering, School of

Medicine, Kermanshah Uni-

versity of Medical Sciences

(KUMS), Kermanshah, Iran

${ }^{5} \mathrm{MD}$, Department of Radiol-

ogy, School of Medicine,

Tehran University of

Medical Sciences (TUMS),

Tehran, Iran

${ }^{6} \mathrm{MD}$, Advanced Diagnostic

and Interventional Radiol-

ogy Research Research

Centre (ADIR), Tehran Uni-

versity of Medical Sciences

(TUMS), Tehran, Iran

${ }^{7} \mathrm{MSc}$ Student, Department

of Medical Physics and

Biomedical Engineering,

School of Medicine, Tehran

University of Medical Sci-

ences (TUMS), Tehran, Iran

*Corresponding author:

Nader Riyahi Alam

Department of Medical

Physics and Biomedical

Engineering, School of

Medicine, Tehran Univer-

sity of Medical Sciences

(TUMS), Tehran, Iran

E-mail: riahinad@sina.

tums.ac.ir

Received: 19 November 2018 Accepted: 29 December 2018 
sorbed by light-absorbing molecules is used to interpret the amount of light attenuated as a criterion for determining the intensity changes of light-absorbing molecules. Therefore, the ability to answer the hemodynamic response of the brain can be extracted [1-4]. Using this functional imaging technology makes it possible to record the changes in the concentration of $\mathrm{HbH}$ and non-oxygenated ( $\mathrm{HbR}$ ) molecules in the blood as hemodynamic response parameters $[5,6]$. The fNIRS technology uses a special wavelengths range of infrared light; between 700 and $1000 \mathrm{~nm}[7,8]$.

Functional imaging using the near-infrared spectrophotometer has special advantages that are inherently suitable for use in studies that are aimed at investigating the cortical response to complex motor stimulation $[2,3]$. These features allow complex motor patterns study which can't be easily implemented using imaging methods such as fMRIs due to close structure.

Near-infrared spectroscopy is a functional imaging-approved technique. Compared to other imaging methods, near-infrared spectroscopy is non-invasive, inexpensive, portable, and has low sensitivity. Compared to EEG, the main advantage of fNIRS is when optodes are mounted on the head; in addition, the signals are resistant to motion and electrical noise. Although fNIRS has a low spatial resolution [9-11], it can be used to differentiate between areas such as areas of movement of hands and feet $[12,13]$.

The mode of imagined motion is defined as the mental image of a motion, without apparent activity occurring in the motor organs $[14,15]$.

Mental exercises can improve motor performance by imagining motion $[16,17]$. The concept of motion imaging, which is based on the assumption of the motion in individuals, can activate the same brain regions in performing [18-21]. Several studies have been conducted on the theory that the movement of body organs activates the same regions in the brain in comparison to the movement of the hands and feet. The primary sensorimotor cortex (PSMC) and the premotor cortex (PMC) are the motion regions of the brain and located near the skull tissue in which are easily accessible for measurement by optical methods.

The cortical regions participating in motor activity and operations are optically recognized as accessible areas. Based on the data obtained from electrophysiological and neuroimaging, the brain is known as a self-organizing organ that its different parts are, however, anatomically distinct, electrical, chemical, hemodynamical, and metabolic processes are effectively interconnected [22,23].

Due to the introduction of functional imaging techniques, fMRI has been widely used in studies of brain activity patterns in the brain cortex [24-31]. Various studies have shown that the neural networks involved in the action-imagination of motion are in the primary motor cortex (M1) and the secondary motor cortex such as the premotor cortex (PMC), and supplementary motor area (SMA) [32]. These neural networks are activated when one learns motor activity through performing (traditional learning), observing (visual learning), and imagining motion [33]. Many studies have shown fNIRS is a reliable method for measuring brain oxygenation associated with the imagination of performing motion $[34,35]$.

A study by Frey and colleagues showed during the observation, imagination, and execution of finger tapping activated premotor cortex, pre-supplementary motor area, and posterior parietal cortex [36]. Researchers, in a study, showed overlapping activity maps were observed through the observation, imagination, and movement of the arm in the areas of the dorsal premotor cortex, superior parietal lobe, and intraparietal sulcus.

Determining the patterns of brain activity, during the implementation of the activity or the imagination of performing a movement, is a hot topic of neuroscience and neuro-rehabilitation. This information can be used for stud- 
ies on the motor cortex in normal people and the mechanisms for rehabilitation in people seeking brain damage, as well as for introducing new rehab strategies in people with brain damage [37,38].

This study aims to analyze the activity of the cerebral cortex during the right wrist motion using fNIRS data. This research was done to achieve patterns of changes in cerebral cortex hemodynamic activity using the results of the infrared spectrophotometer approach and the distinction among brain activity patterns.

\section{Material and Methods}

\section{Participants}

This novel observational Optical Imaging study aims to investigate, ten healthy righthanded young people who participated in this study. The age range was between 25-40 years old. These people were completely healthy in terms of neurology and had no history or symptoms of neurological disease. Before the start of the test, participants were given a detailed description of how to complete this study. In this project, all stages of the work were completed with full knowledge of the participants and received written consent from them. Moreover, all the tests carried out in this study were approved by the Ethics Committee of Tehran University of Medical Sciences (Approval Number: IR.TUMS.MEDICINE REC.1396.3968). Figure 1 shows the schematic chart of various steps in this project.

fNIRS data acquisition

Participants were asked to sit on a hand-held chair opposite the monitor to conduct the test.

Figure 1: A schematic chart of various steps in process of wrist motion direction coding.

The required data for this study were collected using 48-channel fNIRS compatible MR (OxyMonfNIRS from Artinis) with two wavelengths of 845 and $763 \mathrm{~nm}$.

The changes in brain oxygen levels were used to determine the position of the activated area of the brain during the assumption of a right-hand movement. Multi-channel systems computed the changes of Oxy-Hb, Deoxy-Hb, and total-Hb, using the Beer-Lambert law.
The physiological basis for using fNIRS to measure brain activity is the interaction between neuronal activity and subsequent changes in the hemodynamic properties of the brain, known as "neuromuscular coupling" [39,40]. To collect data, 8 transmitters and 8 infrared light receivers were divided into two groups (each with 4 transmitters and 4 receivers) they formed 20 channels overall (Figure 2).

In imaging using fNIRS, determining the 

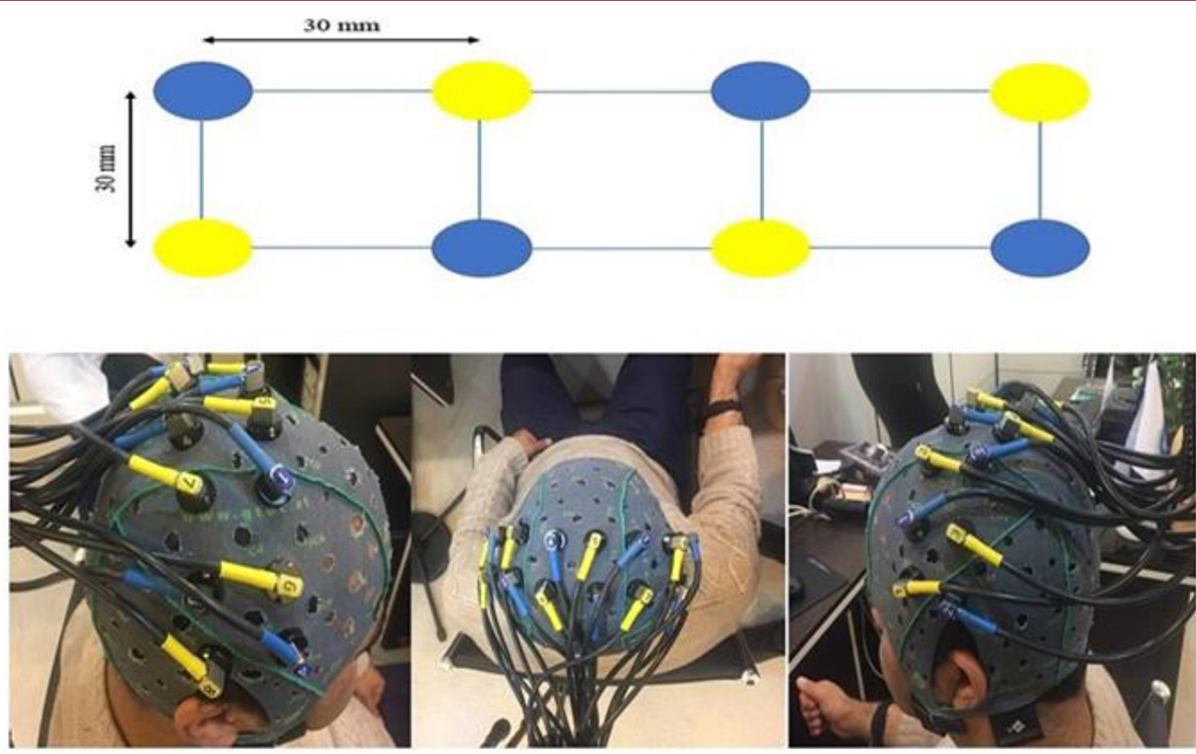

Figure 2: This figure shows that how optodes (receivers and transmitters) should be placed on people's skulls. You can see it from several views. As you see, they should be placed on parietal lobes of the brain and cover all the regions of the motor cortex (blue color represents transmitter and yellow color represents receiver).

depth of light penetration is a major issue, light has a limited penetrating depth within the brain, and this depth of penetration is determined by the distance between the optode.

The greater distance between the receivers and transmitters causes the greater penetration depth of the light, but the intensity of the return light is sharply reduced $[41,42]$. The optimal distance between the optodes is $2-4 \mathrm{~cm}$ [43]. In this test, the distance between optodes was $30 \mathrm{~mm}$ to collect fNIRS data.

An international system (10-20) was used to determine the location of fNIRS optodes on the skull of individuals.

\section{Task Procedure}

Participants had to follow a wrist motion pattern without any movement. The participants were asked to look at the wrist pattern following the directions shown on the screen of the monitor and follow directions up, down, left, and right. They should not move their wrists and only imagine the movement by the direction displayed on the monitor. To achieve this goal, the images (Figure 3) were shown to individuals, and one of the directions, which was randomly illuminated, made the person imagine motion in that direction every time. At the same time as the direction was turned off, the center image became clear, in which the person was in a natural state. The image referred to any particular direction was illuminated for 3 seconds, the person should move to that particular direction during this time; next, the image of the center was illuminated for 2-4 seconds, the person should not be directed (should return to base position). Thus, it took 5-7 seconds to imagine each step of the movement.

Our motion task started with a 4-second rest and included 10 blocks while each block containing 10 steps in different directions, and finally, there was a 4-second rest at the end of each block. The whole test lasted 496 seconds, and during that time, 20 times, each angle was completely referred to randomly (Figure 4). 
Brain Cortical Imagining by fNIRS

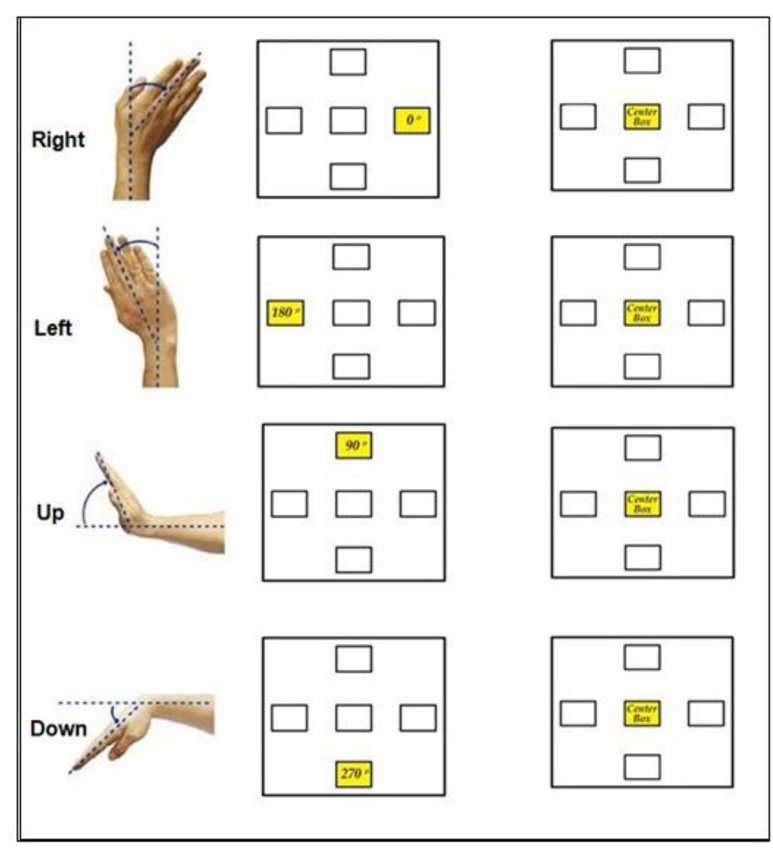

Figure 3: A schematic figure of right wrist motion imagination task. Participants in this test had to follow a wrist motion pattern in up, down, left, and right directions without any movement. They should not move their wrists and only imagined the movement under the direction displayed on the monitor.
Data Analysis

The data was analyzed using NIRS software -statistical parametric mapping (NIRS-SPM) that runs in MATLAB software. The t-statistic SPM maps were calculated at a significant level of $5 \%$. Because $\mathrm{HbO}$ is a sensitive marker indicating hemodynamic changes during neuronal activity, $\mathrm{HbO}$ levels were used to calculate cortical activity to analyze fNIRS data. To correct the generated noise by the fNIRS device, a Gaussian smoothing filter was used. During the data analysis process, the waveletMDL based detrending algorithm was used to correct the fNIRS signaling of respiration, heart rate, and motion. A stand-alone application was used to record the location of fNIRS channels on a standard brain template provided by the Montreal Neurological Institute (Figure 5) [44,45].

\section{GLM Based Analysis}

In this study, we used the GLM model to process fNIRS data. The GLM method is one of the models which is based on the fNIRS data analysis model, in which a data model is initially considered, and after data analysis. It

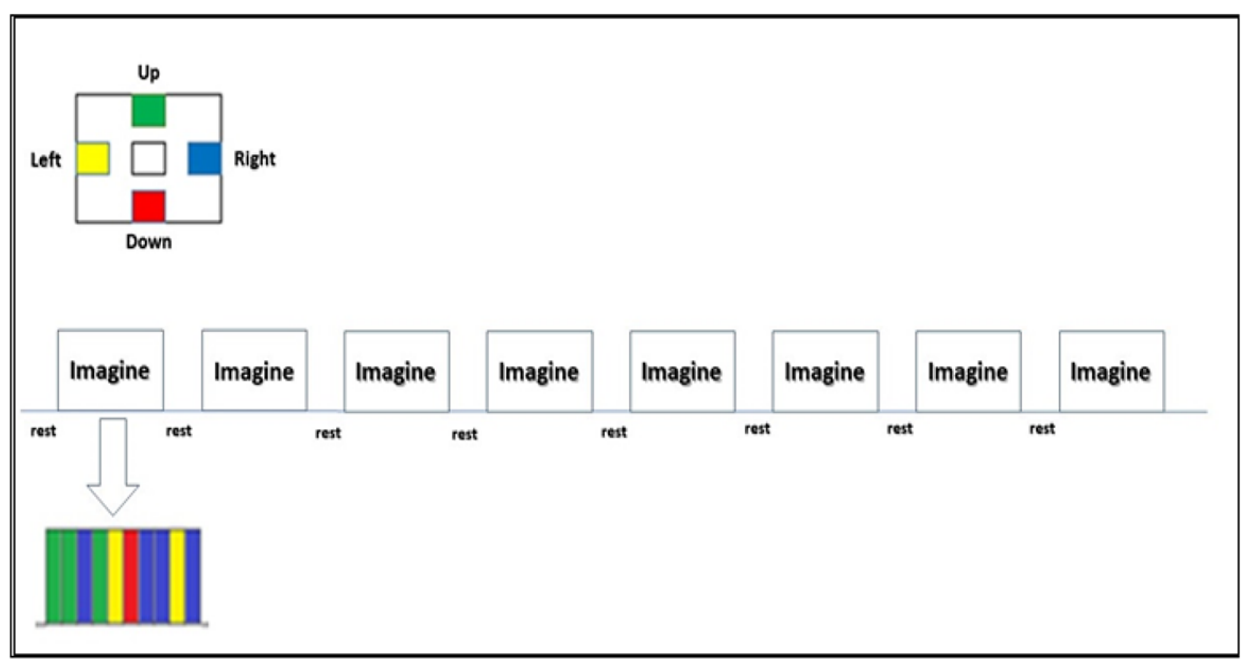

Figure 4: Designing task block of wrist motion imagination. Each of these colors showing a special direction repetition during the process of motor task conduction in which the person should under the displayed direction, imagine it without any movement. The motor pattern which is used contains 8 blocks and each block contains 10 movements. 


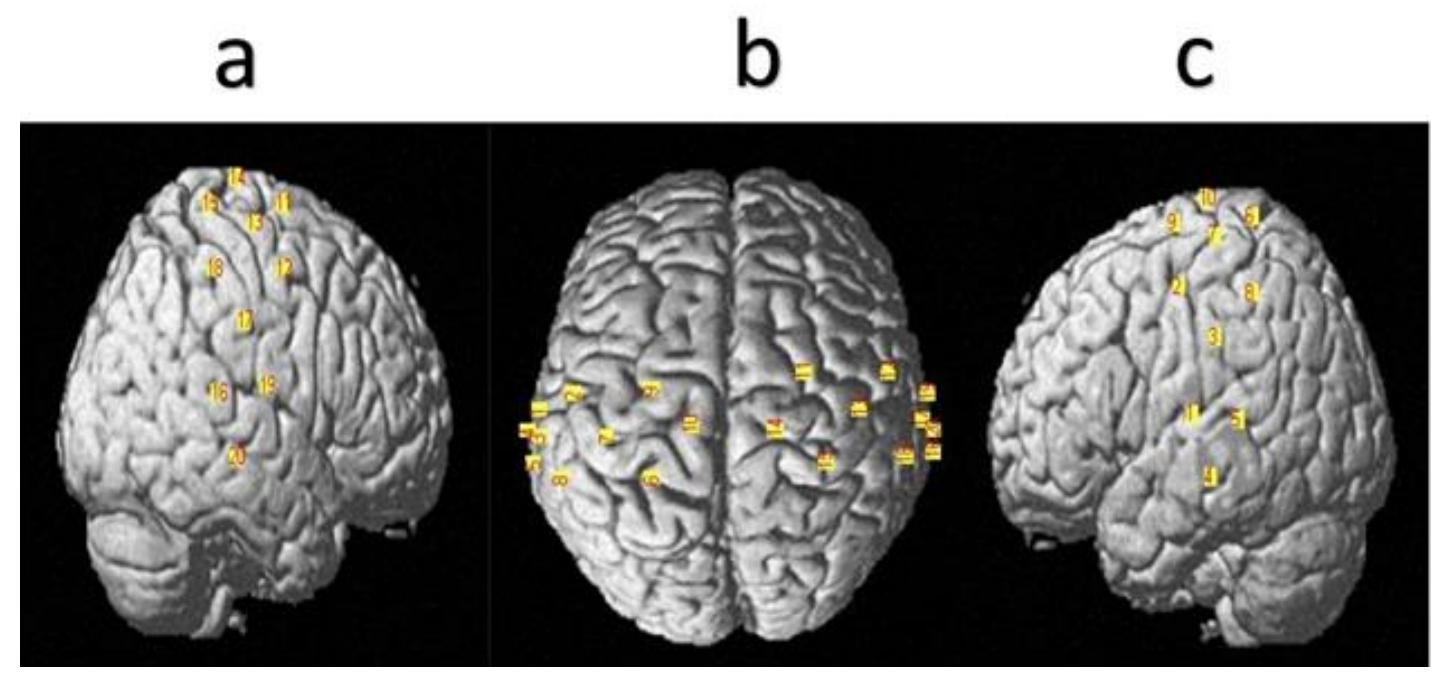

Figure 5: Recording of canals situation in standard Montreal Neurological Institute (MNI) using Stand-alone application. a) canals situation on the standard brain format at Right lateral view b) canals situation on the standard brain format at Dorsal view c) canals situation on the standard brain format at Left lateral view.

is concluded that how much the assumed model is consistent with the data. In this method, a specific model for the excitation function is considered.

It can be believed that if the data and model are matched the data is stimulated by that function.

This linear model is considered as the following.

$$
\mathbf{y}(\mathbf{t})=\boldsymbol{\beta} \mathbf{x}(\mathbf{t})+\mathbf{e}(\mathbf{t})
$$

In which $y(t), x(t), \beta$, and e are the time series of each voxel, the function of the excitation model of each voxel, the estimated parameter for $\mathrm{x}(\mathrm{t})$, and the model error, respectively (Table 1).

\section{Results}

The group analysis of imaging the motion of the wrist to the right showed the activation (a meaningful increase of $\mathrm{HbO}$ ) was divergently extended in the Primary Motor Cortex, Pre-Motor, Supplementary Motor Cortex, and Primary Somatosensory Cortex areas. The analysis of data on the imaging motion of the wrist to the left indicated focal concentration of $\mathrm{HbO}$ was divergent in the Primary Motor Cortex region.

The data analysis of imaging motion of the wrist to downward showed activation (a significant increase in $\mathrm{HbO}$ ) was divergent in the Primary Motor Cortex and Primary Somatosensory Cortex areas.

The results of the group analysis of the present fNIRS data showed directional activities are different and distinct spatially during the motion imagination of the right wrist. Moreover, it's possible to differentiate different directions of hand movement.

Figure 6 shows in two modes, the motion of the wrist to up and left, only Primary Motor Cortex was activated, but in two other modes, in addition to M1, other areas of the cerebral cortex were divergently activated.

The results showed the activation was stronger when it was imagined to move to the right, and more areas of the motor cortex were activated in this case than the other three modes of motion imagination; in addition, a wider neural network was involved in the brain activity.

The activation associated with the imagina- 
Brain Cortical Imagining by fNIRS

Table 1: Montreal Neurological Institute (MNI) coordinates of Functional Near Infrared Spectroscopy (fNIRS) Channel

\begin{tabular}{ccccc} 
Channel Position on MNI ATLAS & X & Y & Z & Number of Channel \\
\hline Superior Temporal Gyrus & -68 & -17 & 11 & 1 \\
\hline Primary Motor Cortex & -56 & -12 & 53 & 2 \\
\hline Primary Somatosensory Cortex & -68 & -28 & 35 & 3 \\
\hline Middle Temporal Gyrus & -71 & -26 & -8 & 4 \\
\hline Superior Temporal Gyrus & -70 & -39 & 10 & 5 \\
\hline Primary Somatosensory Cortex & -28 & -44 & 74 & 6 \\
\hline Primary Motor Cortex & -43 & -27 & 68 & 7 \\
\hline Supramarginal gyrus part of Wernicke's area & -60 & -45 & 51 & 8 \\
\hline Pre-Motor and Supplementary Motor Cortex & -28 & -10 & 73 & 9 \\
\hline Primary Motor Cortex & -13 & -25 & 79 & 10 \\
\hline Pre-Motor and Supplementary Motor Cortex & -26 & 3 & 72 & 11 \\
\hline Pre-Motor and Supplementary Motor Cortex & 57 & -4 & 52 & 12 \\
\hline Primary Motor Cortex & 46 & -18 & 66 & 13 \\
\hline Primary Motor Cortex & 17 & -27 & 79 & 14 \\
\hline Primary Somatosensory Cortex & 35 & -39 & 72 & 15 \\
\hline Superior Temporal Gyrus & 72 & -34 & 14 & 16 \\
\hline Primary Somatosensory Cortex & 69 & -22 & 36 & 17 \\
\hline Supramarginal gyrus part of Wernicke's area & 63 & -36 & 52 & 18 \\
\hline Superior Temporal Gyrus & 70 & -11 & 16 & 19 \\
\hline Middle Temporal Gyrus & 73 & -26 & -6 & 20
\end{tabular}

MNI: Montreal Neurological Institute

tion of motion at 0 and 90 degrees was more in the sides of the brain motor cortex.

The activation associated with motion imagination in directions of 180 and 270 degrees was more in the middle of the M1. The data analysis obtained from the fNIRS functional imaging technique showed in the case of the assumption of performing right-hand movement, there was a significant difference in maximum brain activation focus among the four main directions (up, down, left, and right).

In the imagination of motion upward, significant increasing activation was observed in the BA4. In the imagination of the right-hand movement to downward BA4, BA2 and BA1 were activated. In motion imagination to the right $\mathrm{BA} 4, \mathrm{BA} 6$, and $\mathrm{BA} 1$ were activated, and in the mode of motion imagination to the left
BA4 was activated (Table 2).

\section{Discussion}

The present study aims to investigate the ability of near-infrared spectroscopy imaging (fNIRS) and evaluate cortical activity in the wrist movement imagination, a movement that human beings perform routinely. fNIRS, as a functional optical imaging technique, allows cerebral cortex hemodynamic changes to be observed as a criterion for indirect evaluation of neuronal activity in the brain.

To achieve this goal, we monitored the changes in the $\mathrm{HbO}$ concentration along with the imagination of the right wrist movement in the motor cortex by the fNIRS multichannel machine. We measured the changes in $\mathrm{HbO}$ as a marker for measuring brain activity. $\mathrm{HbO}$ is 


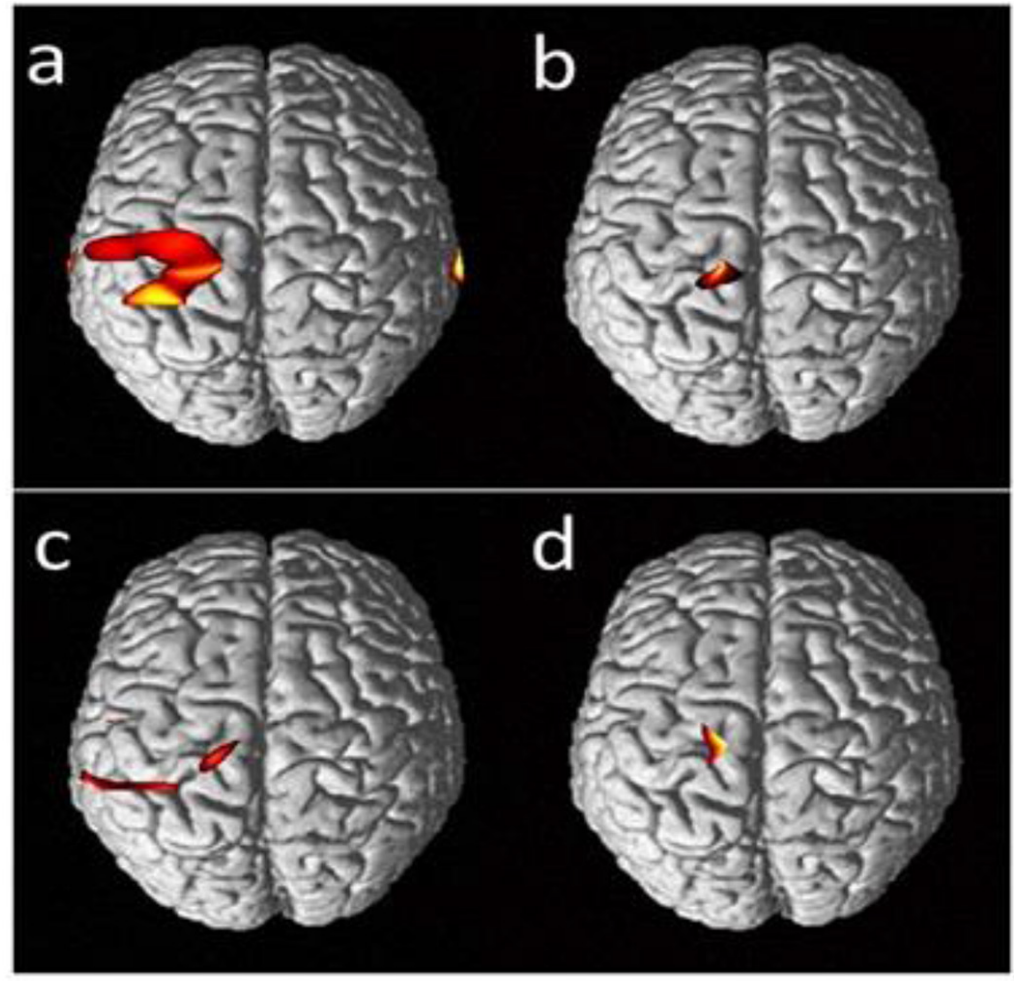

Figure 6: The results obtained from the analysis group from Near-Infrared Spectroscopy-statistical parametric mapping (NIRS_SPM). Map activation obtained from motion imagination of right wrist in the cerebral motor cortex. a) Activation map obtained from motion imagination of right wrist to up b) Activation map obtained from motion imagination of right wrist to down c) Activation map obtained from motion imagination of right wrist to the right d) Activation map obtained from motion imagination of right wrist to the left.

Table 2: Parameters of brain activity during right wrist activity imagination in Functional NearInfrared Spectroscopy (fNIRS).

\begin{tabular}{|c|c|c|c|c|c|c|}
\hline \multirow{2}{*}{$\begin{array}{l}\text { Wrist Movement } \\
\text { Direction }\end{array}$} & \multirow{2}{*}{ Brain Region } & \multirow{2}{*}{ Brodmann Area } & \multicolumn{3}{|c|}{ MNI Position } & \multirow{2}{*}{ Channel Number } \\
\hline & & & $\mathbf{z}$ & $y$ & $\mathbf{x}$ & \\
\hline Up & Primary Motor Cortex & 4 & 79 & -25 & -13 & 10 \\
\hline \multirow{3}{*}{ Down } & Primary Motor Cortex & 4 & 79 & -25 & -13 & 10 \\
\hline & Primary Somatosensory Cortex & 2 & 51 & -45 & 60 & 8 \\
\hline & Primary Somatosensory Cortex & 1 & 74 & -44 & -28 & 6 \\
\hline \multirow{5}{*}{ Right } & Primary Motor Cortex & 4 & 79 & -25 & -13 & 10 \\
\hline & $\begin{array}{l}\text { Pre-Motor and Supplementary } \\
\text { Motor Cortex }\end{array}$ & 6 & 73 & -10 & -28 & 9 \\
\hline & Primary Motor Cortex & 4 & 68 & -27 & -43 & 7 \\
\hline & Primary Somatosensory Cortex & 1 & 74 & -44 & -28 & 6 \\
\hline & Primary Motor Cortex & 4 & 68 & -27 & -43 & 2 \\
\hline Left & Primary Motor Cortex & 4 & 79 & -25 & -13 & 10 \\
\hline
\end{tabular}

MNI: Montreal Neurological Institute 
a parameter widely which is used in fNIRS studies and an indirect measure of brain neural activity by calculating hemodynamic intensity changes in the cerebral cortex (in fact, this parameter shows the amount of oxygen used by neurons) $[46,47]$. The group analysis results showed a significant increase in $\mathrm{HbO}$ concentration in the Primary Motor Cortex and Primary Somatosensory Cortex regions during the right wrist movement to downward. In two modes, imaginations of moving to left and upward were recorded only in the Primary Motor Cortex, and in the mode of motion imagination to the right; in addition, the activated areas in the previous two modes, both Pre-Motor and Supplementary Motor Cortex areas were activated.

Hideki Nakano [48], in his study, showed that in the imagination of chopstick and hammer movements, the regions of the premotor cortex and primary somatosensory cortex were activated, which is consistent with the results of this study.

Various studies have shown during imagination of movement to inferior dorsolateral, prefrontal cortex, inferior frontal gyrus, premotor cortex, primary somatosensory cortex, and primary motor cortex were activated [49-51]. The results showed the activation pattern obtained from moving to the left direction is very similar to the activation pattern in moving upward. The extent of the activation recorded in the imagination of wrist movement to the right was the most valuable in comparison with the other modes, which could be due to the fact a wider neural network plays a role in this case more than the other states (Figure 1).

The design of this motor pattern to examine the activity of the cerebral cortex during the imagination of wrist motion showed the neurons in the motor cortex are involved in controlling and processing the movements in one of the directions of right, left, up, and down. Our findings in this paper showed using fNIRS neural imaging data is a possibility of differentiation among brain activity patterns in different modes of wrist motion imagination.

The major limitation we encountered in this study was the selection of participants who were completely neurologically healthy and had no history or indication of neurological disease. Due to the inherent nature of the light and its low penetration depth into the brain, it is difficult to record changes in brain activity and hemodynamics in the deeper layers of the cerebral cortex. Thus, signal amplifier methods should be used.

\section{Conclusion}

We also found out $\mathrm{HbO}$ concentration in the cerebral cortex increased $(\mathrm{p}<0.05)$ significantly in the mode of imagination of motion relative to the resting state. A significant increase in $\mathrm{HbO}$ concentration in our data supports our knowledge of control and imagination of motion and increases our knowledge of cortical responses during imagining motion patterns.

We believe the results of our study can be a step forward in the field of motion control studies and also provide a pathway for future research on brain activity and clinical trials in people with brain damage. Obtained signals from the data collected in this study can be used to control rehabilitation tools to help people with motion sickness in the future.

\section{Acknowledgment}

The authors wish to thank the Tehran University of Medical Sciences for the financial and instrumental support of this research, and the National Brain Mapping Laboratory (NBML), Tehran, Iran for their assistance during data acquisition.

\section{Conflict of Interest}

\section{None}

\section{References}

1. Gibson AP, Hebden JC, Arridge SR. Recent advances in diffuse optical imaging. Phys Med Biol. 2005;50:R1-43. PubMed PMID: 15773619.

2. Hoshi $Y$. Functional near-infrared spectroscopy: current status and future prospects. Journal of 
Maziar Jalalvandi, et al

Biomedical Optics. 2007;12:062106.

3. Lloyd-Fox S, Blasi A, Elwell CE. Illuminating the developing brain: the past, present and future of functional near infrared spectroscopy. Neurosci Biobehav Rev. 2010;34:269-84. doi: 10.1016/j. neubiorev.2009.07.008. PubMed PMID: 19632270.

4. Strangman G, Boas DA, Sutton JP. Non-invasive neuroimaging using near-infrared light. Biol Psychiatry. 2002;52:679-93. doi: 10.1016/s00063223(02)01550-0.

5. Pellicer A, Bravo Mdel C. Near-infrared spectroscopy: a methodology-focused review. Semin Fetal Neonatal Med. 2011;16:42-9. doi: 10.1016/j. siny.2010.05.003. PubMed PMID: 20580625.

6. León-Carrión J, León-Domínguez U. Functional near-infrared spectroscopy (fNIRS): Principles and neuroscientific applications. Neuroimaging-Methods, Peter Bright, IntechOpen; 2012.

7. Kober SE, Wood G, Kurzmann J, Friedrich EV, Stangl M, Wippel T, et al. Near-infrared spectroscopy based neurofeedback training increases specific motor imagery related cortical activation compared to sham feedback. Biol Psychol. 2014;95:21-30.

8. Villringer A, Chance B. Non-invasive optical spectroscopy and imaging of human brain function. Trends Neurosci. 1997;20:435-42. doi: 10.1016/s0166-2236(97)01132-6. PubMed PMID: 9347608.

9. Boas DA, Dale AM, Franceschini MA. Diffuse optical imaging of brain activation: approaches to optimizing image sensitivity, resolution, and accuracy. Neuroimage. 2004;23 Suppl 1:S275-88. doi: 10.1016/j.neuroimage.2004.07.011. PubMed PMID: 15501097.

10. Cui X, Bray S, Bryant DM, Glover GH, Reiss AL. A quantitative comparison of NIRS and fMRI across multiple cognitive tasks. Neuroimage. 2011;54:2808-21. doi: 10.1016/j.neuroimage.2010.10.069. PubMed PMID: 21047559 PubMed PMCID: PMC3021967.

11. Shibasaki H. Human brain mapping: hemodynamic response and electrophysiology. Clin Neurophysiol. 2008;119:731-43. doi: 10.1016/j. clinph.2007.10.026. PubMed PMID: 18187361.

12. Blankertz B, Tomioka R, Lemm S, Kawanabe M, Muller K-R. Optimizing spatial filters for robust EEG single-trial analysis. IEEE Signal processing magazine. 2008;25:41-56. doi: 10.1109/ msp.2008.4408441.

13. Chin ZY, Ang KK, Wang C, Guan C, Zhang H. Multi-class filter bank common spatial pattern for four-class motor imagery $\mathrm{BCl}$. Conf Proc IEEE Eng Med Biol Soc. 2009;2009:571-4. doi: 10.1109/
IEMBS.2009.5332383. PubMed PMID: 19963466.

14. Wriessnegger S, Kurzmann J, Neuper C. Spatiotemporal differences in brain oxygenation between movement execution and imagery: a multichannel near-infrared spectroscopy study. Int J Psychophysiol. 2008;67:54-63. doi: 10.1016/j.ijpsycho.2007.10.004.

15. Guillot A, Di Rienzo F, Macintyre T, Moran A, Collet C. Imagining is Not Doing but Involves Specific Motor Commands: A Review of Experimental Data Related to Motor Inhibition. Front Hum Neurosci. 2012;6:247. doi: 10.3389/fnhum.2012.00247. PubMed PMID: 22973214. PubMed PMCID: PMC3433680.

16. Guillot A, Hoyek N, Louis M, Collet C. Understanding the timing of motor imagery: recent findings and future directions. International Review of Sport and Exercise Psychology. 2012;5:3-22. doi: 10.1080/1750984x.2011.623787.

17. Faralli A, Bigoni M, Mauro A, Rossi F, Carulli D. Noninvasive strategies to promote functional recovery after stroke. Neural Plast. 2013;2013:854597. doi: 10.1155/2013/854597. PubMed PMID: 23864962. PubMed PMCID: PMC3707231.

18. Garrison KA, Winstein CJ, Aziz-Zadeh L. The mirror neuron system: a neural substrate for methods in stroke rehabilitation. Neurorehabil Neural Repair. 2010;24:404-12. doi: 10.1177/1545968309354536. PubMed PMID: 20207851.

19. Guillot A, Di Rienzo F, Collet C. The neurofunctional architecture of motor imagery. Advanced Brain Neuroimaging Topics in Health and DiseaseMethods and Applications: Intech0pen; 2014.

20. Lacourse MG, Cohen MJ, Lawrence KE, Romero $\mathrm{DH}$. Cortical potentials during imagined movements in individuals with chronic spinal cord injuries. Behav Brain Res. 1999;104:73-88. PubMed PMID: 11125744.

21. Hanakawa T, Dimyan MA, Hallett M. Motor planning, imagery, and execution in the distributed motor network: a time-course study with functional MRI. Cereb Cortex. 2008;18:2775-88. doi: 10.1093/cercor/bhn036.

22. Buzsaki G. Rhythms of the Brain. New York: Oxford University Press; 2006.

23. Palva JM, Palva S. Infra-slow fluctuations in electrophysiological recordings, blood-oxygenationlevel-dependent signals, and psychophysical time series. Neuroimage. 2012;62:2201-11. doi: 10.1016/j.neuroimage.2012.02.060.

24. Ehrsson $H H$, Fagergren $A$, Jonsson $T$, Westling $G$, Johansson RS, Forssberg $\mathrm{H}$. Cortical activity in 
precision- versus power-grip tasks: an fMRI study. J Neurophysiol. 2000;83:528-36. doi: 10.1152/ jn.2000.83.1.528. PubMed PMID: 10634893.

25. Kapreli E, Athanasopoulos S, Papathanasiou M, Van Hecke P, Strimpakos N, Gouliamos A, et al. Lateralization of brain activity during lower limb joints movement. An fMRI study. Neuroimage. 2006;32:1709-21. doi: 10.1016/j.neuroimage.2006.05.043. PubMed PMID: 16859927.

26. Kapreli E, Athanasopoulos S, Papathanasiou M, Van Hecke P, Keleki D, Peeters R, et al. Lower limb sensorimotor network: issues of somatotopy and overlap. Cortex. 2007;43:219-32. doi: 10.1016/s0010-9452(08)70477-5. PubMed PMID: 17405668 .

27. Kim MJ, Hong JH, Jang SH. The cortical effect of clapping in the human brain: A functional MRI study. NeuroRehabilitation. 2011;28:75-9. doi: 10.3233/NRE-2011-0634. PubMed PMID: 21447906.

28. laPointe KE, Klein JA, Konkol ML, Kveno SM, Bhatt $\mathrm{E}$, DiFabio RP, et al. Cortical activation during finger tracking vs. ankle tracking in healthy subjects. Restor Neurol Neurosci. 2009;27:253-64. PubMed PMID: 19813287.

29. Luft AR, Smith GV, Forrester L, Whitall J, Macko RF, Hauser TK, et al. Comparing brain activation associated with isolated upper and lower limb movement across corresponding joints. Hum Brain Mapp. 2002;17:131-40. doi: 10.1002/hbm.10058. PubMed PMID: 12353246.

30. Rao SM, Bandettini PA, Binder JR, Bobholz JA, Hammeke TA, Stein EA, et al. Relationship between finger movement rate and functional magnetic resonance signal change in human primary motor cortex. J Cereb Blood Flow Metab. 1996;16:12504. doi: 10.1097/00004647-199611000-00020. PubMed PMID: 8898698.

31. Wexler BE, Fulbright RK, Lacadie CM, Skudlarski P, Kelz MB, Constable RT, et al. An fMRI study of the human cortical motor system response to increasing functional demands. Magn Reson Imaging. 1997;15:385-96. PubMed PMID9223039.

32. Lotze M, Montoya P, Erb M, Hulsmann E, Flor H, Klose $\mathrm{U}$, et al. Activation of cortical and cerebellar motor areas during executed and imagined hand movements: an fMRI study. J Cogn Neurosci. 1999;11:491-501. PubMed PMID: 10511638.

33. Rizzolatti G, Fadiga L, Gallese V, Fogassi L. Premotor cortex and the recognition of motor actions. Brain Res Cogn Brain Res. 1996;3:131-41. doi: 10.1016/0926-6410(95)00038-0. PubMed PMID: 8713554.
34. Fuchino $Y$, Nagao $M$, Katura T, Bando M, Naito $M$, Maki A, et al. High cognitive function of an ALS patient in the totally locked-in state. Neurosci Lett. 2008;435:85-9. doi: 10.1016/j.neulet.2008.01.046. PubMed PMID: 18359565.

35. Kanoh Si, Murayama Y-m, Miyamoto K-i, Yoshinobu T, Kawashima R, editors. A NIRS-based braincomputer interface system during motor imagery: system development and online feedback training. Annual International Conference of the IEEE Engineering in Medicine and Biology Society; Minneapolis, USA: IEEE; 2009. p. 594-7. doi: 10.1109/ iembs.2009.5333710.

36. Macuga KL, Frey SH. Neural representations involved in observed, imagined, and imitated actions are dissociable and hierarchically organized. Neuroimage. 2012;59:2798-807. doi: 10.1016/j.neuroimage.2011.09.083. PubMed PMID: 22005592. PubMed PMCID: PMC3254825.

37. Kaplan MS. Plasticity after brain lesions: contemporary concepts. Arch Phys Med Rehabil. 1988;69:984-91. PubMed PMID: 3056323.

38. York DH. Review of descending motor pathways involved with transcranial stimulation. Neurosurgery. 1987;20:70-3. doi: 10.1097/00006123198701000-00021. PubMed PMID: 3543726.

39. Vanzetta I, Grinvald A. Coupling between neuronal activity and microcirculation: implications for functional brain imaging. HFSP J. 2008;2:79-98. doi: 10.2976/1.2889618. PubMed PMID: 19404475. PubMed PMCID: PMC2645573.

40. Kleinfeld D, Mitra PP, Helmchen F, Denk W. Fluctuations and stimulus-induced changes in blood flow observed in individual capillaries in layers 2 through 4 of rat neocortex. Proceedings of the National Academy of Sciences. 1998;95:15741-6. doi: 10.1073/pnas.95.26.15741.

41. Dirnagl U, Villringer A, Gebhardt R, Haberl RL, Schmiedek P, Einhäupl KM. Three-dimensional reconstruction of the rat brain cortical microcirculation in vivo. J Cereb Blood Flow Metab. 1991;11:353-60. doi: 10.1038/jcbfm.1991.74.

42. Colier W, Quaresima V, Oeseburg B, Ferrari M. Human motor-cortex oxygenation changes induced by cyclic coupled movements of hand and foot. Exp Brain Res. 1999;129:457-61. doi: 10.1007/ s002210050913.

43. Obrig H, Wenzel R, Kohl M, Horst S, Wobst P, Steinbrink J, et al. Near-infrared spectroscopy: does it function in functional activation studies of the adult brain? Int J Psychophysiol. 2000;35:125-42. doi: 10.1016/s0167-8760(99)00048-3. PubMed PMID: 10677642. 
44. Tak S, Yoon SJ, Jang J, Yoo K, Jeong Y, Ye JC. Quantitative analysis of hemodynamic and metabolic changes in subcortical vascular dementia using simultaneous near-infrared spectroscopy and fMRI measurements. Neuroimage. 2011;55:17684. doi: 10.1016/j.neuroimage.2010.11.046.

45. Ye JC, Tak S, Jang KE, Jung J, Jang J. NIRSSPM: statistical parametric mapping for near-infrared spectroscopy. Neuroimage. 2009;44:428-47. doi: 10.1016/j.neuroimage.2008.08.036. PubMed PMID: 18848897.

46. Steinbrink J, Villringer A, Kempf F, Haux D, Boden $\mathrm{S}$, Obrig H. Illuminating the BOLD signal: combined fMRI-fNIRS studies. Magn Reson Imaging. 2006;24:495-505. doi: 10.1016/j.mri.2005.12.034. PubMed PMID: 16677956.

47. Huppert TJ, Diamond SG, Franceschini MA, Boas DA. HomER: a review of time-series analysis methods for near-infrared spectroscopy of the brain. Applied optics. 2009;48:D280-98. doi: 10.1364/ a0.48.00d280.
48. Nakano H, Ueta K, Osumi M, Morioka S. Brain activity during the observation, imagery, and execution of tool use: an fNIRS/EEG study. $J$ Novel Physiother S. 2012;1. doi: 10.4172/2165-7025. s1-009.

49. Grezes J, Decety J. Functional anatomy of execution, mental simulation, observation, and verb generation of actions: a meta-analysis. Hum Brain Mapp. 2001;12:1-19.doi:10.1002/1097-0193(200101)12:1<1:.aidhbm10>3.0.c0;2-v.

50. Lorey B, Pilgramm S, Bischoff M, Stark R, Vaitl $D$, Kindermann $S$, et al. Activation of the parieto-premotor network is associated with vivid motor imagery-a parametric fMRI study. PLOS One. 2011;6:e20368. doi: 10.1371/journal. pone. 0020368 .

51. Solodkin A, Hlustik P, Chen EE, Small SL. Fine modulation in network activation during motor execution and motor imagery. Cereb Cortex. 2004;14:1246-55. doi: 10.1093/cercor/bhh086. 